\title{
Single-Crystal Neutron Structural Analyses of Potassium Dihydrogen Phosphate and Potassium Dideuterium Phosphate
}

\author{
Tatsuki MIYOSHI*, Hiroyuki MASHIYAMA, Takanao ASAHI, Hiroyuki KIMURA ${ }^{1}$, and Yukio NODA ${ }^{1}$ \\ Faculty of Science, Yamaguchi University, Yamaguchi 753-8512, Japan \\ ${ }^{1}$ Institute of Multidisciplinary Research for Advanced Materials, Tohoku University, Sendai 980-8577, Japan
}

\begin{abstract}
Single-crystal neutron structural analyses have been performed on both potassium dihydrogen phosphate (KDP) and potassium dideuterium phosphate (DKDP) in order to discuss the isotope effect from structural viewpoints. The values of spontaneous polarization are calculated from the refined structural parameters by the point-charge method, and the calculated values are almost in good agreement with the experimental values of each compound. The temperature dependences of the anisotropic atomic displacement parameters $U_{33}$ 's and positional shifts of potassium, phosphorus, oxygen, and hydrogen/deuterium atoms along the polar $c$-axis is compared between KDP and DKDP. It is concluded that the paraelectricferroelectric transitions are perfectly of the improper order-disorder type in both KDP and DKDP; the ordering of the hydrogen atom induces the spontaneous displacements of both potassium and phosphorus atoms. The interatomic distances and angles in the paraelectric and ferroelectric phases of KDP and DKDP are also investigated. From the result, all the structural differences seem to be caused by the difference in mass between protons and deuterons.
\end{abstract}

KEYWORDS: hydrogen-bond-type ferroelectrics, proton and deuteron, ferroelectric phase transition, single-crystal structural analysis, neutron diffraction

\section{Introduction}

Potassium dihydrogen phosphate $\left(\mathrm{KH}_{2} \mathrm{PO}_{4}\right.$, hereafter abbreviated to KDP) is one of the well-known ferroelectrics discovered by Busch and Scherrer. ${ }^{1)}$ The crystal is easily grown from a water solution by the slow evaporation method, stable in atmospheric condition, useful in piezoelectric and nonlinear optic devices, and well known as a typical ferroelectric with a Curie temperature $T_{\mathrm{c}}=123 \mathrm{~K}$, where the paraelectricferroelectric phase transition takes place. Its paraelectric and ferroelectric phases have body-centered tetragonal $(I \overline{4} 2 d$, $\left.D_{2 d}^{12}, Z=4\right)$ and face-centered orthorhombic $\left(F d d 2, C_{2 v}^{19}\right.$, $Z=8$ ) systems, respectively, and its ferroelectricity appears along the $c$-axis. KDP is often categorized in KDP family ferroelectrics or hydrogen-bond-type ferroelectrics, in which a proton in an $\mathrm{O}-\mathrm{H} \cdots \mathrm{O}$ bond is considered to play an important role in ferroelectric phase transition. ${ }^{2)}$

The deuterated crystal potassium dideuterium phosphate $\left(\mathrm{KD}_{2} \mathrm{PO}_{4}\right.$, hereafter abbreviated to DKDP) is isomorphous to KDP, and its paraelectric-ferroelectric phase transition takes place at $c a .220 \mathrm{~K}$. Both KDP and DKDP come up frequently in discussions about KDP-type ferroelectrics together, as if they are permanently inseparable counterparts. They are known for the deuteration effect or isotope effect showing that the Curie temperatures of their deuteron compounds are $\mathrm{ca} .100 \mathrm{~K}$ higher than their proton compounds. ${ }^{3)}$

In the paraelectric (tetragonal) phase of KDP, a proton occupies two sites related by a symmetry operation with equal probability. The disordered distribution of the proton between two oxygen atoms of the hydrogen bond in KDP was confirmed by neutron diffraction study. ${ }^{4)}$ Each $\mathrm{PO}_{4}$ tetrahedron is linked to four adjacent $\mathrm{PO}_{4}$ tetrahedra by hydrogen bonds, which lie along the $a$ - or $b$-direction of the tetragonal cell

*E-mail address: struct@yamaguchi-u.ac.jp perpendicular to the ferroelectric $c$-axis. The O-to-O distance $R_{\mathrm{O}-\mathrm{O}}$ linked by the proton is $c a .2 .5 \AA$, and the distance $r$ between the oxygen atom and the nearby proton is $c a$. $1 \AA$. In the ferroelectric phase (orthorhombic phase), a proton attaches to an acceptor oxygen atom. Accordingly, the symmetry is lowered, and the ordered proton is observed directly also by single-crystal neutron diffraction study. ${ }^{5}$ )

It is well known that only two protons attach to a $\mathrm{PO}_{4}$ tetrahedron, which is known as the ice rule. ${ }^{2)}$ In the ferroelectric phase, the distance between two acceptors (named O1) decreases slightly, and the distance between two donors (named O2) increases slightly, in comparison with that in the tetragonal phase. The symmetry of the $\mathrm{H}_{2} \mathrm{PO}_{4}{ }^{-}$cluster is $C_{2}$, and the $S_{4}$ symmetry of the $\mathrm{PO}_{4}$ tetrahedron should be lowered at low temperatures. ${ }^{5)}$

Although the structural change from the paraelectric phase to the ferroelectric phase is accepted by every researcher, the dynamical mechanism of the ferroelectric phase transition in KDP is not conclusive yet. Blinc took the quantum effect in the order-disorder transition of the proton configuration into account, while assuming proton tunneling to explain the isotope effect. ${ }^{6)}$ Other researchers followed this proton tunneling model, and then gave some theoretical works including the model. $^{7-10)}$

Ichikawa and coworkers noticed geometrical isotope effects among hydrogen-bond-type ferroelectrics and their deuterated isomorphs; the hydrogen-bond lengths seem to have unique systematic differences between hydrogen and deuterium compounds. ${ }^{1-13)}$ More concretely, the distance $R_{\mathrm{O}-\mathrm{O}}$ is about $0.05 \AA$ longer in DKDP than in KDP. This longer $R_{\mathrm{O}-\mathrm{O}}$ induces ordering at high temperatures, which is the key to understand the isotope effects in KDP family crystals.

Moreover, Tominaga et al. claimed that the tunneling model is inadequate since no evidence of the softening of the 
proton tunneling mode has been detected directly. ${ }^{14)}$ Additionally, they found forbidden Raman lines in the paraelectric phase, which indicated that the point symmetry of a $\mathrm{PO}_{4}$ tetrahedron was $C_{2}$, the orthorhombic symmetry, but not the tetragonal $S_{4} \cdot{ }^{15}$ ) Therefore, they proposed an order-disordertype transition mechanism on the basis of a mean structure including the locally and momentarily distorted $\mathrm{PO}_{4}$ tetrahedron. ${ }^{14-17)}$

On the other hand, the quantum motion of protons within a double-well potential has been analyzed by many authors. The tunneling frequencies, if they exist independently, are estimated at $c a$. 500 and $50 \mathrm{~cm}^{-1}$ in KDP and DKDP, respectively. ${ }^{10,18-21)}$

The crystal structures of KDP and DKDP around the paraelectric-ferroelectric phase transition temperature were refined by Nelmes and his coworkers. ${ }^{22-27)}$ The main results are as follows:

(i) In the paraelectric phase, the density distribution of protons has two peaks separated by a distance $\delta$, which is about $0.32 \AA$ in KDP or $0.45 \AA$ in DKDP just above $T_{\mathrm{c}}$.

(ii) In the ferroelectric phase, the proton distribution becomes an unbalanced one just below $T_{\mathrm{c}}$, and finally appears as a single peak at the acceptor $\mathrm{O} 1$ side.

(iii) With decreasing temperature, the atomic displacement parameter decreases linearly in the paraelectric phase. However, the $U_{33}$ of the phosphorus atom has a positive cut point in the zero-temperature extrapolation, which may indicate the disorder of the phosphorus atom along the polar axis in the paraelectric phase.

The coherent neutron scattering used in structural analysis reflects only the mean structure of the crystal, so that it is difficult to distinguish between a dynamic disorder and a static or quasi-static disorder. The former may be called a quantum tunnel, while the latter a classical disorder. In order to see the dynamical motion of protons directly, incoherent neutron scattering experiments were performed by Ikeda et al., who refuted proton tunneling. ${ }^{28)}$ Therefore, the order-disorder transition mechanism not of a proton tunneling but of a disordered $\mathrm{PO}_{4}$ tetrahedron was accepted widely.

The pressure effect on $T_{\mathrm{c}}$ is also well-known. ${ }^{29,30)}$ Samara proposed an empirical criterion to specify the character of the phase transition in ferroelectrics; the transition temperature increases with the application of hydrostatic pressure if the transition is of the order-disorder type, while for displacivetype ferroelectrics the temperature dependence is reversed. ${ }^{29)}$ It has been noted that the ferroelectric phase transition temperature of both KDP and DKDP decreases with the application of hydrostatic pressure, which is in contrast to typical substances that exhibit an order-disorder type transition. Moreover, the ferroelectric phase transition disappears down to zero temperature at the respective critical pressures, around which the dielectric constant shows a quantum paraelectric character, as if the transition is displacive. ${ }^{30)}$

Such confusing situations were resolved partially. In 2002, Reiter et al. carefully observed the incoherent scattering from $\mathrm{KDP}$ and found evidence of proton tunneling, i.e., protons are coherent over the two sites in the paraelectric phase. ${ }^{31)}$

First-principles calculations have been developed to investigate rather complex systems such as KDP and DKDP. ${ }^{32)}$ It was revealed that structural transition accompanies electronic charge redistribution and ionic displacement as a consequence of proton ordering. Moreover, proton tunneling is coupled with heavier ions that exhibit cluster collective motion, but not single-particle dynamics. A simplified model of KDP also explained the displacive character of KDP on the basis of the tunneling motion of a 'particle' with half of the effective mass of the $\mathrm{H}_{2} \mathrm{PO}_{4}{ }^{-}$cluster. ${ }^{33)}$

The refined structural parameters of ferroelectric phases in the low-temperature region were reported at $T_{\mathrm{c}}-20 \mathrm{~K}$ and $T_{\mathrm{c}}-10 \mathrm{~K}$ for KDP and DKDP, respectively, by Nelmes et $a l^{25)}$ To the authors' knowledge, structural studies of KDP are limited down to the liquid nitrogen temperature, and those of DKDP down to $210 \mathrm{~K}$. In addition to the structural parameters in the paraelectric phase, those in the ferroelectric phase are effective and important in the comprehensive understanding of the phase transition mechanism.

Therefore, we perform the single-crystal neutron diffraction experiments of both KDP and DKDP in a wide temperature range (down to $10 \mathrm{~K}$ ) for the ferroelectric and paraelectric phases and refine the structure parameters, in order to examine the essential difference between KDP and DKDP. The experimental process is described in $\$ 2$. Results of the structure refinement are presented in $\$ 3$, and discussed in $\$ 4$. Finally, conclusions are presented in $\S 5$.

\section{Experimental Process}

\subsection{Single-crystal specimens}

An experimental specimen of KDP with rectangular dimensions of $3.1 \times 3.2 \times 2.1 \mathrm{~mm}^{3}$ (along the tetragonal $a-, b-$, and $c$ - axes) was cut from a transparent region of a grown parent crystal provided by Dr. K. Gesi. The ferroelectric transition temperature was $123 \mathrm{~K}$.

A single crystal of DKDP was grown by the slow evaporation method from a heavy water solution kept in a desiccator at room temperature for about two weeks. The reagent with a deuteration ratio of $98 \%$ was supplied from Cambridge Isotope Laboratories, Inc., and heavy water with a deuteration ratio of $99.9 \%$ from ISOTEC, commercially. As an experimental specimen, a block with rectangular dimensions of 2.8 $\times 2.6 \times 2.5 \mathrm{~mm}^{3}$ (along the tetragonal $a-, b$-, and $c$-axes) was cut from a transparent region of the grown parent crystal. The deuterium replacement ratio of the crystal was estimated to be $95.8 \%$ from the peak position of the dielectric constant at $214 \mathrm{~K}^{14)}$

The surfaces of the specimens were polished carefully using filter paper wet with distilled or heavy water. As an electrode, silver paste or evaporated aluminum was attached on the $c$-planes of the specimens, and DC bias fields of 240$360 \mathrm{~V} / \mathrm{mm}$ were applied by a power supply (Glassman High Voltage INC.) in order to keep the single domain states only in the ferroelectrics phases of KDP and DKDP. The specimens were glued on vanadium sticks using insulating varnish (GE low-temperature varnish).

\subsection{Single-crystal neutron diffraction experiment}

Single-crystal neutron diffraction experiments were performed using a four-circle-off-center-type diffractometer, FONDER $^{34)}$ (T2-2) of JRR-3M, JAEA, Tokai. The neutron wavelengths in those experiments were 1.2452 and $1.2396 \AA$ 
Table I. Measured temperatures $[\mathrm{K}]$, numbers of independent reflections, and residual factors ( $R_{1}$-factors) [\%] in KDP and DKDP.

\begin{tabular}{cccccc}
\hline $\begin{array}{c}\text { KDP } \\
\text { Temp. }\end{array}$ & No. Refs. & $R_{1}$ & $\begin{array}{c}\text { DKDP } \\
\text { Temp. }\end{array}$ & No. Refs. & $R_{1}$ \\
\hline 200 & 197 & 3.90 & 298 & 222 & 3.19 \\
150 & 215 & 3.28 & 250 & 220 & 4.24 \\
125 & 208 & 2.73 & 220 & 219 & 4.23 \\
120 & 388 & 3.54 & 210 & 366 & 4.64 \\
118 & 390 & 3.51 & 205 & 398 & 4.73 \\
115 & 390 & 3.27 & 190 & 373 & 5.06 \\
110 & 380 & 3.22 & 160 & 397 & 4.83 \\
70 & 374 & 3.44 & 130 & 403 & 4.55 \\
30 & 382 & 3.81 & 70 & 390 & 4.57 \\
10 & 375 & 3.26 & 10 & 381 & 5.16 \\
\hline
\end{tabular}

for KDP and DKDP, respectively, and the diffraction angle was $2 \theta<156^{\circ}$.

The temperature of the specimens was controlled using a TEMCON system of the cryostat accessory of FONDER. The diffraction experiments were performed at ten temperatures for each compound: 10, 30, 70, 110, 115, 118, 120, 125, 150, and $200 \mathrm{~K}$ for KDP, and 10, 70, 130, 160, 190, 205, 210, 220, 250 , and $298 \mathrm{~K}$ for DKDP. About 220 and 420 unique reflections were measured in the paraelectric and ferroelectric phases, respectively.

\subsection{Data analysis}

Firstly, each reflection profile was checked, and the net intensity and standard deviation were reduced using a Profile Check program of FONDER. Absorption and extinction corrections were applied to the analysis of the raw diffraction data using the programs of DABEXN and RADIELN, ${ }^{35,36)}$ and then equivalent reflections of those corrected ones were averaged. The index ranges were $h, k \leq 11, l \leq 10$ and $h, k \leq 15, l \leq 10$ in the paraelectric and ferroelectric phases, respectively.

For the obtained diffraction datasets, the structural parameters were refined using the full-matrix least-squares program SHELXL-97,37) via WinGX. ${ }^{38)}$ The initial parameters of the fractional coordinates of atoms were those reported by Bacon and Pease. ${ }^{4,5)}$ In our analyses, the space groups of the paraelectric and ferroelectric phases are $I \overline{4} 2 d$ and $F d d 2$, respectively, and the standard symmetry coordinates are used. In the final processes of all the structure refinements, anisotropic Debye-Waller factors were used for all the atoms. A splitatom method was applied to the analysis of protons/deuterons in the paraelectric phase. All the least-squares refinements converged, and yielded $R_{1}$-factors of 2.73-3.90 and 3.19$5.16 \%$ for KDP and DKDP, respectively. Here, the $R_{1}$-factor is defined by

$$
R_{1}=\frac{\sum_{\boldsymbol{h}}\left\|F_{\mathrm{o}}(\boldsymbol{h})|-| F_{\mathrm{c}}(\boldsymbol{h})\right\|}{\sum_{\boldsymbol{h}}\left|F_{\mathrm{o}}(\boldsymbol{h})\right|},
$$

where $F_{\mathrm{o}}(\boldsymbol{h})$ and $F_{\mathrm{c}}(\boldsymbol{h})$ are the observed and calculated structure factors for the index $\boldsymbol{h}$, respectively. The measurement conditions and the obtained $R_{1}$-factors are tabulated in Table I.
Table II. Atomic parameters of KDP at $10 \mathrm{~K}$. The unit cell parameters are 10.544(7), 10.481(6), and 6.920(5) $\AA$ for $a, b$, and $c$, respectively. $x, y$, and $z$ are the fractional coordinates, and $U_{\mathrm{eq}}\left[10^{-4} \AA^{2}\right]$ is the isotropic atomic displacement parameter. The values in parentheses are the estimated standard deviations (e.s.d.'s), and the parameters without e.s.d.'s are fixed ones.

\begin{tabular}{ccccc}
\hline Atom & $x$ & $y$ & $z$ & $U_{\mathrm{eq}}$ \\
\hline $\mathrm{K}$ & 0 & 0 & $0.4837(5)$ & $45(4)$ \\
$\mathrm{P}$ & 0 & 0 & 0 & $34(3)$ \\
$\mathrm{O} 1$ & $0.03399(9)$ & $0.11549(9)$ & $-0.1369(3)$ & $53(2)$ \\
$\mathrm{O} 2$ & $0.11617(9)$ & $-0.0344(1)$ & $0.1169(4)$ & $50(2)$ \\
$\mathrm{H}$ & $0.2127(2)$ & $0.0632(2)$ & $0.1138(6)$ & $166(4)$ \\
\hline
\end{tabular}

Table III. Atomic parameters of DKDP at $10 \mathrm{~K}$. The unit cell parameters are 10.595(9), 10.487(6), and 6.922(4) $\AA$ for $a, b$, and $c$, respectively. $x$, $y$, and $z$ are the fractional coordinates, and $U_{\mathrm{eq}}\left[10^{-4} \AA^{2}\right]$ is the isotropic atomic displacement parameter. The values in parentheses are e.s.d.'s, and the parameters without e.s.d.'s are fixed ones.

\begin{tabular}{ccccc}
\hline Atom & $x$ & $y$ & $z$ & $U_{\mathrm{eq}}$ \\
\hline $\mathrm{K}$ & 0 & 0 & $0.4824(6)$ & $44(6)$ \\
$\mathrm{P}$ & 0 & 0 & 0 & $24(4)$ \\
$\mathrm{O} 1$ & $0.0354(1)$ & $0.1149(1)$ & $-0.1373(5)$ & $42(3)$ \\
$\mathrm{O} 2$ & $0.1158(1)$ & $-0.0352(1)$ & $0.1166(5)$ & $40(3)$ \\
$\mathrm{D}$ & $0.2160(2)$ & $0.0651(2)$ & $0.1141(6)$ & $142(4)$ \\
\hline
\end{tabular}

\section{Results}

\subsection{Atomic parameters}

The atomic parameters at $10 \mathrm{~K}$ of KDP and DKDP are shown in Tables II and III, respectively. The lattice parameter $a$ of DKDP is $0.05 \AA$ longer than that of KDP, while the $b$ and $c$ values are almost the same, as described in the table captions.

Here, the descriptions of the atomic parameters at the other temperatures in the ferroelectric phase are excluded because they are almost the same as those at $10 \mathrm{~K}$, except for the atomic displacement parameters. The atomic parameters of the paraelectric phase are also omitted because they are in agreement with those reported previously. ${ }^{22,26,27)}$

\subsection{Interatomic distances and angles}

Hereafter, interatomic distances and angles are compared between KDP and DKDP in figures, where the solid and open symbols represent KDP and DKDP, respectively.

A $\mathrm{K}$ atom is surrounded by eight oxygen atoms; four of them are the nearest-neighbor (NN) atoms, and the other four are the next-nearest-neighbor (NNN) atoms. Below $T_{\mathrm{c}}$, K displaces along the $c$-axis, so that these distances between $\mathrm{K}$ and $\mathrm{O}$ change, as shown in Fig. 1. In the ferroelectric phase, the $\mathrm{NN}$ distance splits into $\mathrm{K}-\mathrm{O} 2_{\mathrm{NN}}$ and $\mathrm{K}-\mathrm{O} 1_{\mathrm{NN}}$, and the $\mathrm{NNN}$ distance into $\mathrm{K}-\mathrm{O} 2_{\mathrm{NNN}}$ and $\mathrm{K}-\mathrm{O} 1_{\mathrm{NNN}}{ }^{26)}$ Strictly, $\mathrm{K}-\mathrm{O} 2_{\mathrm{NN}}$, $\mathrm{K}-\mathrm{O} 1_{\mathrm{NN}}, \mathrm{K}-\mathrm{O} 2_{\mathrm{NNN}}$, and $\mathrm{K}-\mathrm{O} 1_{\mathrm{NNN}}$ indicate $\mathrm{K}-\mathrm{O} 2^{\mathrm{a}}, \mathrm{K}-\mathrm{O} 1^{\mathrm{b}}$, $\mathrm{K}-\mathrm{O} 2$, and $\mathrm{K}-\mathrm{O} 1^{\mathrm{c}}$, respectively; the superscripts $\mathrm{a}, \mathrm{b}$, and $\mathrm{c}$ denote the symmetry coordinates $-x+1 / 4, y+1 / 4, z+1 / 4$; $x-1 / 4,-y+1 / 4, z+3 / 4$; and $x, y, z+1$, respectively.

The $\mathrm{O}-\mathrm{K}-\mathrm{O}$ angles are shown in Fig. 2; the primed and normal O's are NN and NNN ones, respectively. At each angle, $\mathrm{O}$ atoms are related to the two-fold symmetry through the $\mathrm{K}$ atom along the $c$-axis. The four interatomic angles in the ferroelectric phase turn into two interatomic angles in the paraelectric phase, which are crystallographically indepen- 


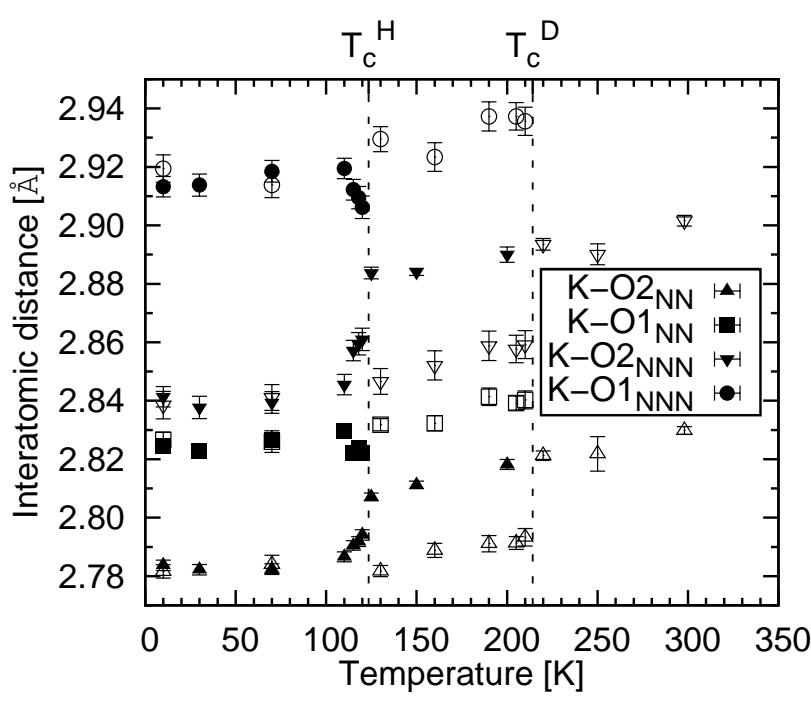

Fig. 1. Temperature dependences of the interatomic distances $\mathrm{K}-\mathrm{O} 2_{\mathrm{NN}}$, $\mathrm{K}-\mathrm{O} 1_{\mathrm{NN}}, \mathrm{K}-\mathrm{O} 2_{\mathrm{NNN}}$, and $\mathrm{K}-\mathrm{O} 1_{\mathrm{NNN}}$. The solid and open symbols denote the values of KDP and DKDP, respectively. 'NN' and 'NNN' mean 'nearest-neighbor' and 'next-nearest-neighbor', respectively. ${ }^{26)}$

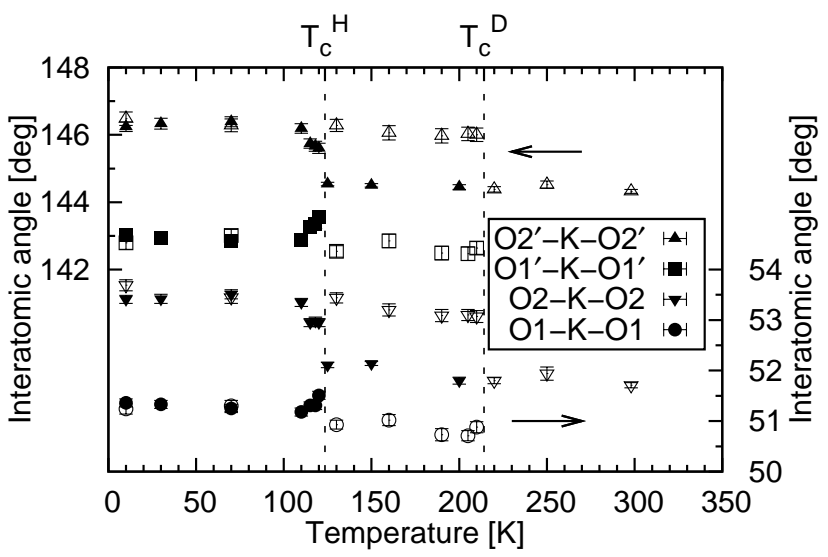

Fig. 2. Temperature dependences of the interatomic angles $\mathrm{O} 2^{\prime}-\mathrm{K}-\mathrm{O} 2^{\prime}$ (left ordinate), $\mathrm{O}^{\prime}-\mathrm{K}-\mathrm{O} 1^{\prime}$ (left ordinate), $\mathrm{O} 2-\mathrm{K}-\mathrm{O} 2$ (right ordinate), and $\mathrm{O} 1-\mathrm{K}-\mathrm{O} 1$ (right ordinate) in both KDP (solid symbols) and DKDP (open symbols).

dent of each other. Strictly, $\mathrm{O} 2^{\prime}-\mathrm{K}-\mathrm{O} 2^{\prime}, \mathrm{O}^{\prime}-\mathrm{K}-\mathrm{O} 1^{\prime}, \mathrm{O} 2-\mathrm{K}-$ $\mathrm{O} 2$, and $\mathrm{O} 1-\mathrm{K}-\mathrm{O} 1$ indicate $\mathrm{O} 2^{\mathrm{a}}-\mathrm{K}-\mathrm{O} 2^{\mathrm{d}}, \mathrm{O} 1^{\mathrm{b}}-\mathrm{K}-\mathrm{O} 1^{\mathrm{e}}, \mathrm{O} 2-$ $\mathrm{K}-\mathrm{O} 2^{\mathrm{f}}$, and $\mathrm{O} 1^{\mathrm{c}}-\mathrm{K}-\mathrm{O} 1^{\mathrm{g}}$, respectively; the superscripts $\mathrm{d}$, e, $\mathrm{f}$, and $\mathrm{g}$ denote the symmetry operations $x-1 / 4,-y-1 / 4, z+1 / 4$; $-x+1 / 4, y-1 / 4, z+3 / 4 ;-x,-y, z ;$ and $-x,-y, z+1$, respectively. Except for the gradual changes just below the $T_{\mathrm{c}}$ of KDP, their interatomic distances and angles change in the same fashion, although the transition temperatures are quite different between KDP and DKDP. With decreasing temperature, the atomic distances slightly decrease as shown in Fig. 1, however, the angles do not show such changes. So, the atomic distance of $\mathrm{K}-\mathrm{O}$ contracts homogeneously within each phase.

Each $\mathrm{K}$ atom is surrounded by six $\mathrm{PO}_{4}$ tetrahedra. Two of them are above and below $\mathrm{K}$ along the $c$-axis, and four around the $c$-axis. The distances between $\mathrm{K}$ and $\mathrm{P}$ for the former neighbors are shorter than for the latter neighbors. The temperature dependences of the $\mathrm{K}-\mathrm{P}$ distances are similar to

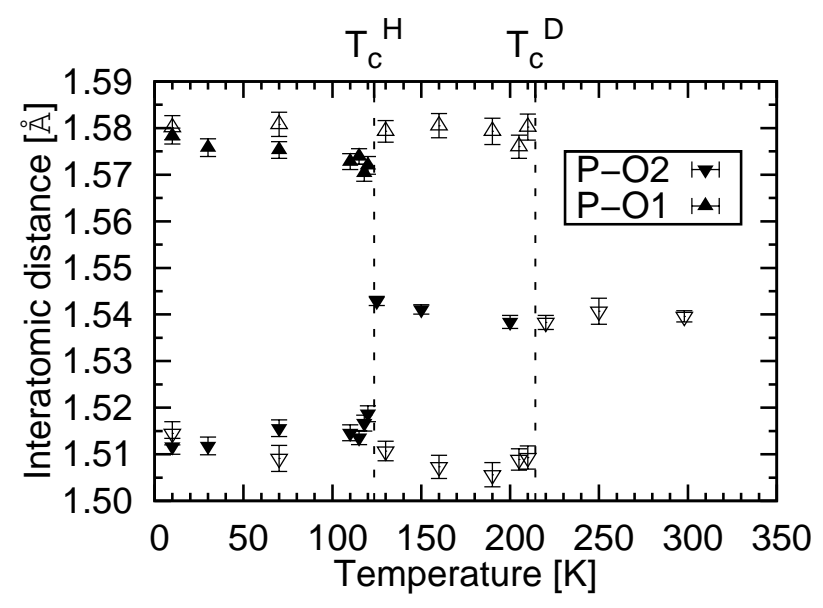

Fig. 3. Temperature dependences of the interatomic distances $\mathrm{P}-\mathrm{O} 2$ and $\mathrm{P}-\mathrm{O} 1$ in both KDP (solid symbols) and DKDP (open symbols).

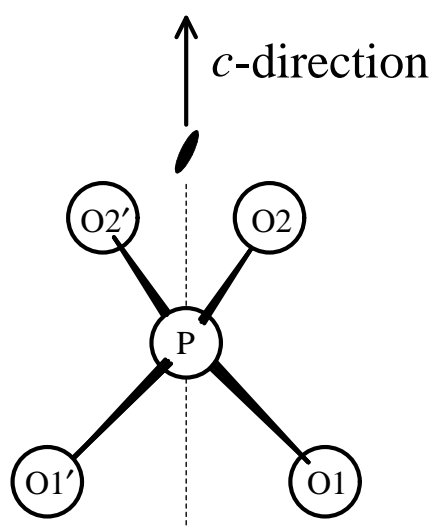

Fig. 4. Schematic view of the $\mathrm{PO}_{4}$ tetrahedron and the two-fold axis along the $c$-axis.

those of $\mathrm{K}-\mathrm{O}$ distances shown in Fig. 1. As shown in Figs. 1 and 2, no notable difference between KDP and DKDP can be recognized in the interatomic distances and angles of $\mathrm{K}-\mathrm{O}$ and $\mathrm{K}-\mathrm{P}$, except for the gradual changes just below the $T_{\mathrm{c}}$ of KDP.

Next, we describe the deformation of the $\mathrm{PO}_{4}$ tetrahedron. In the paraelectric phase, the molecule has an $S_{4}$ symmetry, which is reduced to $C_{2}$ in the ferroelectric phase. Therefore, the unique $\mathrm{P}-\mathrm{O}$ distance splits into $\mathrm{P}-\mathrm{O} 1$ and $\mathrm{P}-\mathrm{O} 2$, where $\mathrm{O} 1$ is the acceptor for the hydrogen atom and $\mathrm{O} 2$ the donor. Figure 3 shows the temperature dependences of the interatomic distances for KDP and DKDP. If the hydrogen atom attaches to $\mathrm{O} 1$, then the $\mathrm{P}-\mathrm{O} 1$ distance increases by $c a$. $0.03 \AA$, while the $\mathrm{P}-\mathrm{O} 2$ distance decreases by $c a$. $0.03 \AA$; the distance change in DKDP is almost the same as or slightly larger than that in KDP. The difference between $\mathrm{P}-\mathrm{O} 2$ and $\mathrm{P}-\mathrm{O} 1$ indicates that the $\mathrm{PO}_{4}$ tetrahedron distorts slightly in the ferroelectrics phase. Except for the gradual changes just below $T_{\mathrm{c}}$ of KDP, the values of both ferroelectric and paraelectric phases in KDP and DKDP are almost the same.

As shown in the schematic picture in Fig. 4, there are two interatomic angles in the paraelectric phase: $\mathrm{O}-\mathrm{P}-\mathrm{O}^{\prime} \sim$ $110.3^{\circ}$ and $\mathrm{O} 1-\mathrm{P}-\mathrm{O} 2 \sim 109.0^{\circ}$. These angles are almost the 


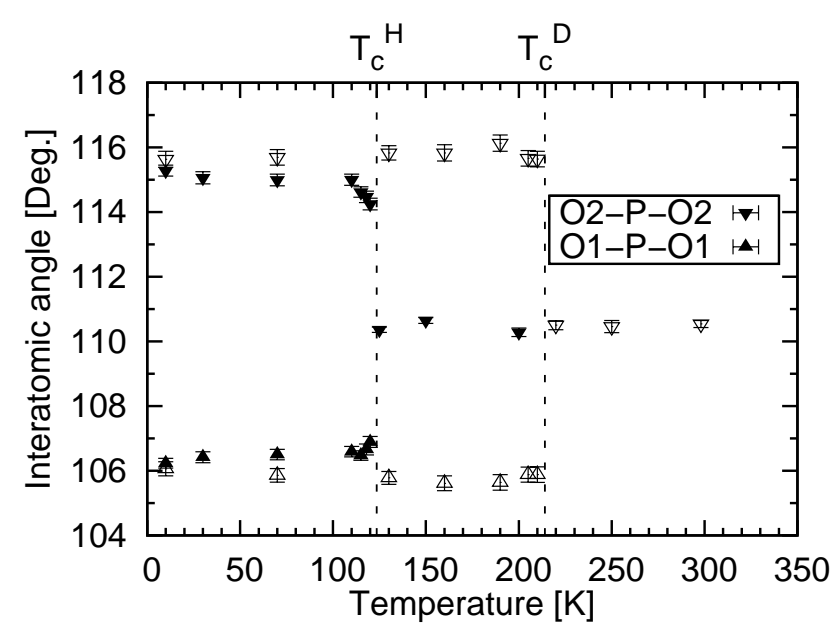

Fig. 5. Temperature dependences of the interatomic angles, $\mathrm{O} 2-\mathrm{P}-\mathrm{O} 2$ and O1-P-O1 in both KDP (solid symbols) and DKDP (open symbols).

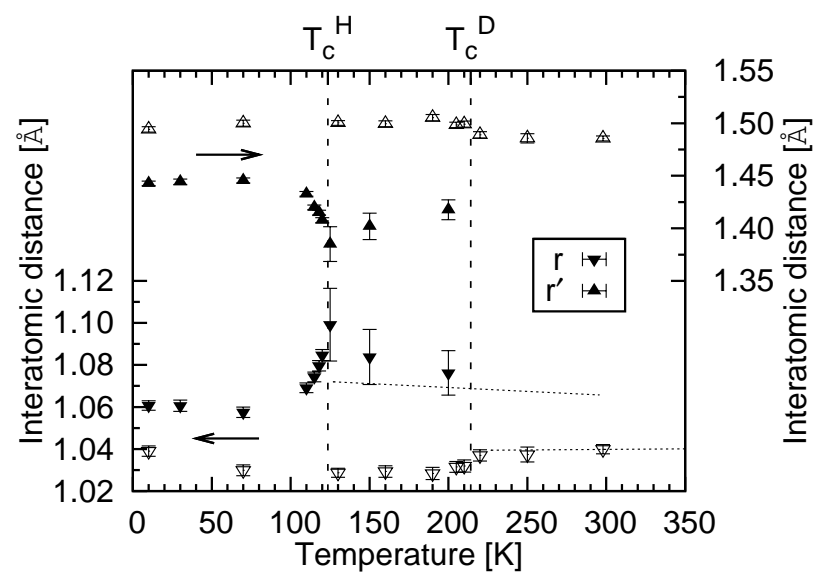

Fig. 6. Temperature dependences of the interatomic distances $r$ (left ordinate) and $r^{\prime}$ (right ordinate) between oxygen and hydrogen. The solid and open symbols denote the values of KDP and DKDP, respectively. Here, $r$ and $r^{\prime}$ are defined as the distances of the $\mathrm{O}-\mathrm{H} / \mathrm{D}$ bond and the H/D $\cdots \mathrm{O}$ bond, respectively. The dotted lines indicate the reported values in the paraelectric phase. ${ }^{22)}$

same for KDP and DKDP. In the ferroelectric phase, the angle $\mathrm{O}-\mathrm{P}-\mathrm{O}^{\prime}$ splits into $\mathrm{O} 1-\mathrm{P}-\mathrm{O} 1^{\prime}$ and $\mathrm{O} 2-\mathrm{P}-\mathrm{O} 2^{\prime}$; the former $\mathrm{O} 1$ atom is the acceptor of $\mathrm{H} / \mathrm{D}$ and the former angle decreases to $106^{\circ}$, and the latter angle increases to $115.5^{\circ}$, as shown in Fig. 5. The deformation of the $\mathrm{PO}_{4}$ tetrahedron in the ferroelectric phase means the following: i) the $\mathrm{O} 1-\mathrm{O} 1^{\prime}$ distance decreases by $c a$. $0.01 \AA$, ii) the $\mathrm{O} 2-\mathrm{O} 2^{\prime}$ distance increases by ca. $0.01 \AA$, and iii) the $\mathrm{P}$ atom shifts along the $z$-axis relatively to the $z$-parameters of $\mathrm{O}$ atoms (getting away from $\mathrm{O} 1$ and approaching $\mathrm{O} 2$ ), while iv) the $\mathrm{O} 1-\mathrm{O} 2$ distance increases slightly. The successive change in i)-iii) is considered to be caused by the ordering of the hydrogen atom (i.e., H/D approaches O1), and is well known as the Cochran mode. ${ }^{39)}$ The deformed $\mathrm{PO}_{4}$ tetrahedron has $C_{2}$ symmetry in the ferroelectric phase; however, the tetrahedron is $S_{4}$ in the paraelectric phase.

Next let us mention the position of H/D. In the ferroelectric phase, the $\mathrm{H}$ atom is at the distances $r$ and $r^{\prime}$ from the ac-

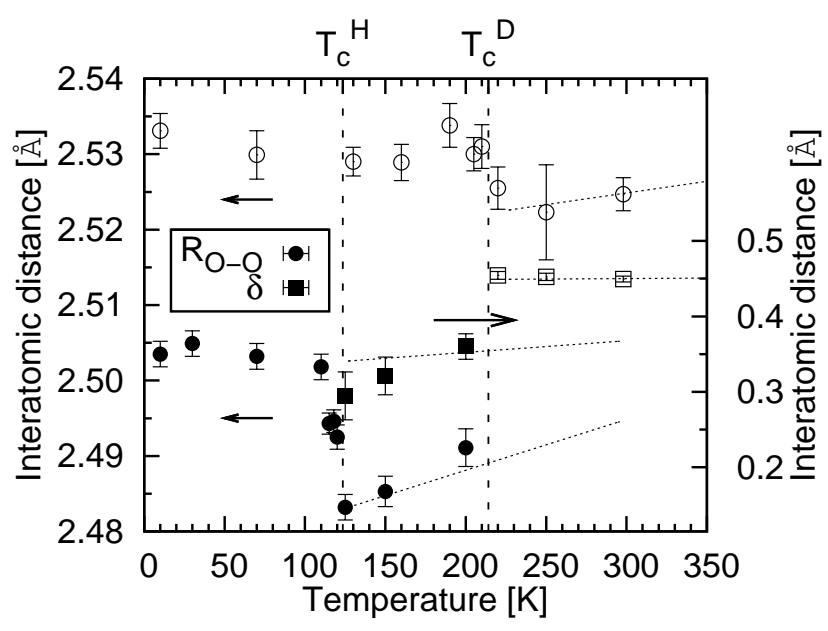

Fig. 7. Temperature dependences of the interatomic distances $R_{\mathrm{O}-\mathrm{O}}$ (left ordinate) and $\delta$ (right ordinate) for the hydrogen bond in both KDP (solid symbols) and DKDP (open symbols). The dotted lines indicate previously reported data. ${ }^{22)}$

ceptor $\mathrm{O} 1$ and the donor $\mathrm{O} 2$, respectively. Above $T_{\mathrm{c}}, r$ and $r^{\prime}$ mean the distances from one of the two disordered positions to the hydrogen-bonded oxygen position. Figure 6 shows the temperature dependences of the distances $r$ (left ordinate) and $r^{\prime}$ (right ordinate). It is apparent that values of $r$ and $r^{\prime}$ are not identical for KDP (solid symbols) and DKDP (open symbols). Although the large e.s.d. (estimated standard deviation) due to the noticeable thermal vibration of protons in KDP may not be negligible, $r$ increases ( $r^{\prime}$ decreases) with decreasing temperature; the same tendency was also reported previously. ${ }^{22)}$ Just below $T_{\mathrm{c}}$ of KDP, $r$ and $r^{\prime}$ show gradual changes. On the other hand, the $r$ and $r^{\prime}$ of DKDP change stepwise at $T_{\mathrm{c}}$, and $r$ coincides with the previously reported data indicated by the dotted line. The $r$ of DKDP is smaller than that of KDP even in the ferroelectric phase.

Figure 7 shows the temperature dependences of the interatomic distances $R_{\mathrm{O}-\mathrm{O}}$ (left ordinate) and $\delta$ (right ordinate) for the hydrogen bond in both KDP (solid symbols) and DKDP (open symbols). $R_{\mathrm{O}-\mathrm{O}}$ and $\delta$ indicate the distances between two oxygen atoms as the donor and acceptor and between two positions at which hydrogen occupies with one-half probability in the paraelectric phase, respectively. ${ }^{22,23,26)}$ The behaviors of $R_{\mathrm{O}-\mathrm{O}}$ in the vicinities of the transition temperatures are also remarkable. For KDP, $R_{\mathrm{O}-\mathrm{O}}$ in the ferroelectric phase is ca. $2.503 \AA$, and decreases as it approaches $T_{c}$. Just above $T_{c}, R_{\mathrm{O}-\mathrm{O}}$ is $c a .2 .483 \AA$, and then increases gradually with a temperature rise in the paraelectric phase. On the other hand, $R_{\mathrm{O}-\mathrm{O}}$ for DKDP remains almost constant within the e.s.d.'s in each phase; a small discontinuous change can be detected at the transition point.

The disordered hydrogen in the paraelectric phase can be represented by the split-atom method, in which each atom occupies two sites with equal probability. The split parameter $\delta$ of KDP depends on temperature slightly, and it is about $0.30 \AA$ just above $T_{\mathrm{c}}$. On the other hand, $\delta=0.45 \AA$ of DKDP is almost temperature-independent. These values of $\delta$ are almost in agreement with those previously reported by Nelmes et al. $^{22)}$ 


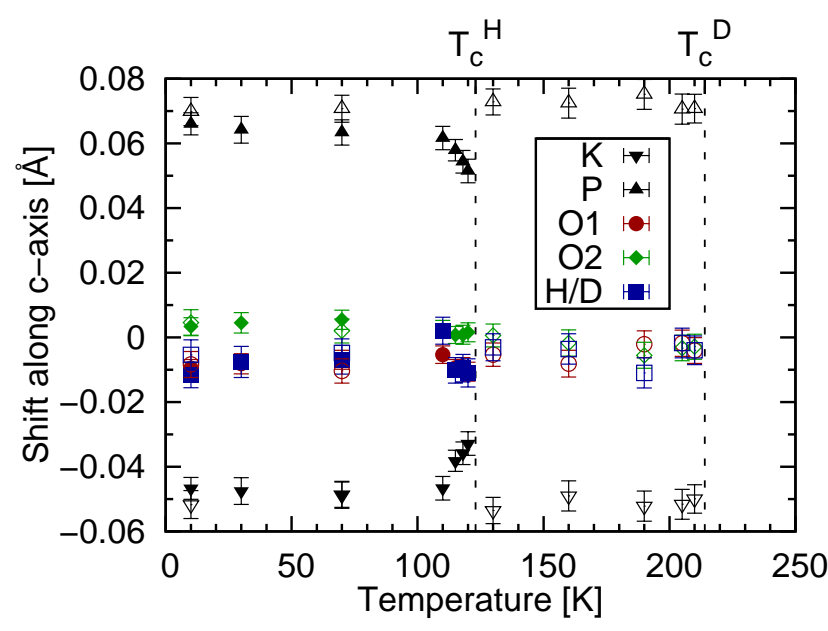

Fig. 8. (Color online) Temperature dependences of the atomic shifts along the polar $c$-axis in the ferroelectric phases of both KDP (solid symbols) and DKDP (open symbols). The positional shifts are calculated as relative displacements in the center-of-gravity coordinate system.

Although the difference in $R_{\mathrm{O}-\mathrm{O}}$ is $c a$. $0.04 \AA$, the difference in $\delta$ between both crystals is remarkably large, amounting to $c a$. $0.1 \AA$. This difference in $\delta$ between KDP and DKDP seems to arise mainly from the dissimilar behaviors of proton and deuteron in the hydrogen bond. Since $R_{\mathrm{O}-\mathrm{O}}$ does not differ markedly, it can be assumed that protons and deuterons exist in approximately equivalent potentials of the hydrogen bonds. Since the proton is a lighter particle than the deuteron, the zero-point energy is higher and the density distribution of protons lies farther from oxygen atoms, which induces a smaller separation of $\delta$ for KDP than for DKDP.

\subsection{Positional shifts and spontaneous polarization along $c$ - axis}

The potassium and phosphorus atoms displace in the opposite direction along the polar $c$-axis after structural phase transition into the ferroelectric phase. Figure 8 shows the temperature dependences of the atomic shifts along the polar $c$-axis in both KDP (solid symbols) and DKDP (open symbols). The positional shifts of all the atoms in the unit cell are directly calculated as relative displacements in the center-of-gravity coordinate system. The center of gravity of the unit cell in the ferroelectric phase coincides with that of the paraelectric phase. To keep the center of gravity of the unit cell, the atomic displacement of the phosphorus atom is made larger than that of the potassium atom because of the difference in the atomic mass. The displacements of the potassium and phosphorus atoms are $c a$. -0.05 and $+0.065 \AA$, respectively. In KDP, these two atoms shift gradually with decreasing temperature, which is different from that observed in DKDP, where the atomic positions are almost constant in the whole ferroelectric phase. The displacements of the potassium and phosphorus atoms in DKDP are larger than those in KDP. On the other hand, the displacements along the $c$-axis of the oxygen and hydrogen/deuterium atoms are overwhelmingly smaller than those of the potassium and phosphorus atoms.

From the atomic shifts along the $c$-axis, we can estimate the polarization. Let us adopt the point-charge method, and

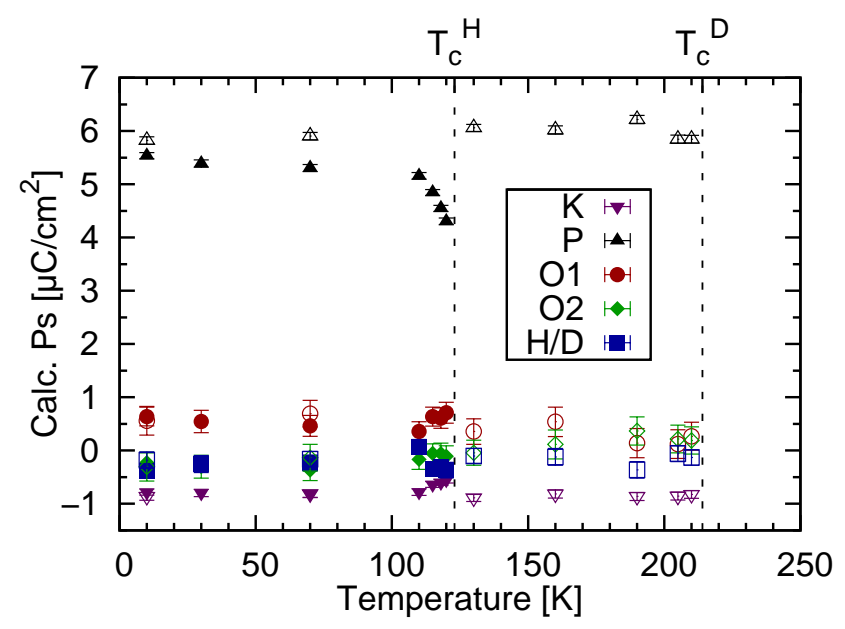

Fig. 9. (Color online) Temperature dependences of the calculated atomic contributions to polarization along the polar $c$-axis in the ferroelectric phases of both KDP (solid symbols) and DKDP (open symbols) by the point-charge method. $+e,+5 e,-2 e$, and $+e$ are assigned as charges of the $\mathrm{K}, \mathrm{P}, \mathrm{O}$, and $\mathrm{H} / \mathrm{D}$ atoms, respectively.

assign $+e,+5 e,-2 e$, and $+e$ as charges of the potassium, phosphorus, oxygen, and hydrogen/deuterium atoms, respectively. The contribution of each atom is calculated using the displacement given in Fig. 8. The temperature dependences of the calculated components of $P_{\mathrm{s}}$ are shown in Fig. 9 for KDP (solid symbols) and DKDP (open symbols). The contribution of the distortion of the $\mathrm{O}_{4}$ frame of the $\mathrm{PO}_{4}$ tetrahedron is not so large. The dominant contributions are the displacements of the phosphorus and potassium atoms along the $c$ axis. If the phosphorus atom shifts in the positive direction, then the potassium atom shifts in the negative direction. The contribution of the phosphorus atom is overwhelmingly larger than that of the potassium atom because the charge of the phosphorus atom is five times larger than that of the potassium atom. Consequently, positive spontaneous polarization appears along the $c$-axis.

The calculated polarizations are compared with the experimental values of KDP and DKDP (deuterium replacement ratio of $98 \%$ ) reported by Samara, ${ }^{40)}$ in Fig. 10 . At around $T_{\mathrm{c}}$, the calculated values are in agreement with the reported ones, which indicates that the point-charge method is well applicable to the study of both KDP and DKDP. In particular, the calculated values in the vicinity of the paraelectric-ferroelectric phase transition in KDP show a gradual change, which is in good agreement with the experimental result. The experimental values reported by Samara and the values calculated by us indicate that the spontaneous polarization of DKDP is $c a$. 1.2 times larger than that of KDP. This is mainly due to the displacement of the phosphorus atom, which is discussed in $\S 4$.

Here, we note the effect of the applied DC bias field. By referring to the X-ray dilatometric study under a DC bias field, ${ }^{41)}$ our field strength is considered to shift $T_{\mathrm{c}}$ slightly, and to be insufficient to induce a qualitative change in the phase transition. 


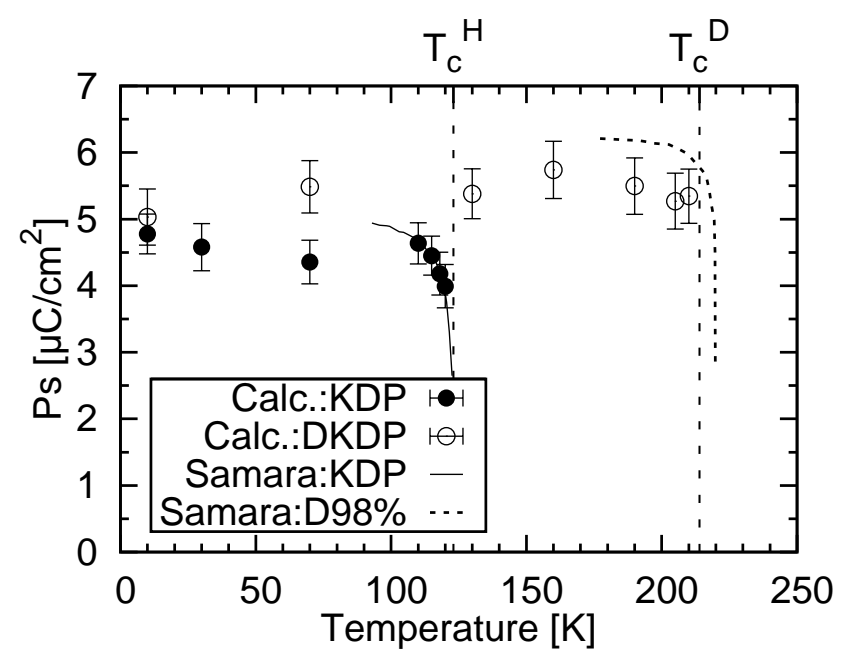

Fig. 10. Temperature dependences of the calculated spontaneous polarization, $P_{\mathrm{s}}$, along the polar $c$-axis in the ferroelectric phases of both KDP (solid symbols) and DKDP (open symbols) by the point-charge method. The solid and broken lines show the experimental values of KDP and DKDP (deuterium replacement ratio of $98 \%$ ), respectively, reported by Samara. ${ }^{40)}$

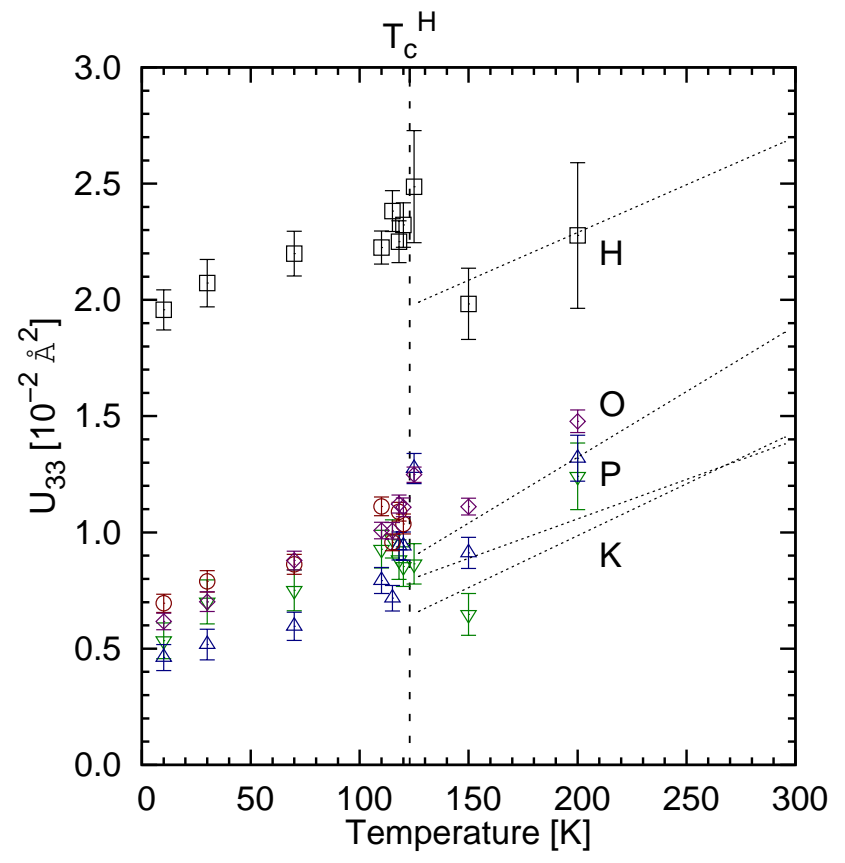

Fig. 11. (Color online) Temperature dependences of the atomic displacement parameters, $U_{33}$ 's, of KDP. The symbols $\nabla, \Delta, \bigcirc, \diamond$, and $\square$ denote the values of the $\mathrm{K}, \mathrm{P}, \mathrm{O} 1, \mathrm{O} 2$, and $\mathrm{H}$ atoms, respectively. The $\mathrm{O} 1$ atom becomes equivalent to the $\mathrm{O} 2$ atom in the paraelectric phase. The dotted lines denote the interpolated temperature dependences from the values at 295 and $127 \mathrm{~K}$ reported by Nelmes et al. ${ }^{22)}$ The error bars indicate e.s.d.'s.

\subsection{Atomic displacement parameter $U_{33}$}

From the least-squares calculations, we obtain the DebyeWaller factor $T(\boldsymbol{h})$ and the atomic displacement parameters:

$$
\begin{aligned}
T(\boldsymbol{h})= & \exp \left[-2 \pi^{2}\left(h^{2} a^{* 2} U_{11}+k^{2} b^{* 2} U_{22}+l^{2} c^{* 2} U_{33}\right.\right. \\
& \left.\left.+2 h k a^{*} b^{*} U_{12}+2 h l a^{*} c^{*} U_{13}+2 k l b^{*} c^{*} U_{23}\right)\right] .
\end{aligned}
$$

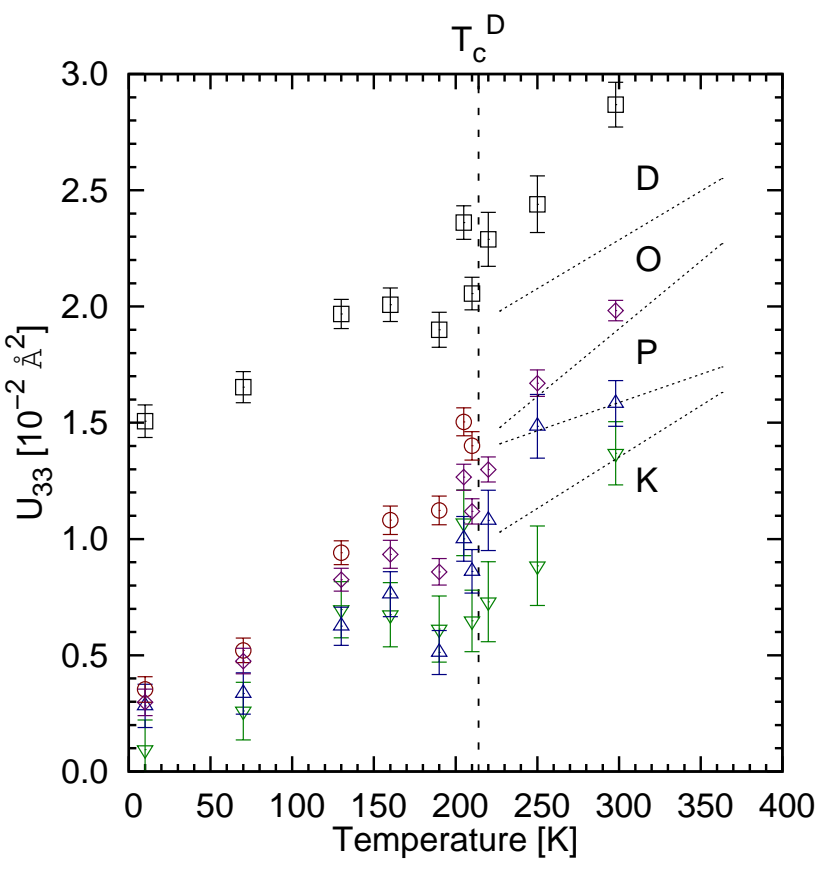

Fig. 12. (Color online) Temperature dependences of the atomic displacement parameters $U_{33}$ 's of DKDP. The symbols $\nabla, \Delta, \bigcirc, \diamond$, and $\square$ denote the values of the $\mathrm{K}, \mathrm{P}, \mathrm{O} 1, \mathrm{O} 2$, and $\mathrm{D}$ atoms, respectively. The $\mathrm{O} 1$ atom becomes equivalent to the $\mathrm{O} 2$ atom in the paraelectric phase. The dotted lines denote the interpolated data at 363 and $227 \mathrm{~K}$ reported by Nelmes et $a .^{22)}$

The atomic displacement parameter is directly related to the mean square displacement if the atom vibrates within a harmonic potential, i.e.,

$$
U_{33}=c^{2}\left\langle(z-\bar{z})^{2}\right\rangle \text {. }
$$

The temperature dependences of $U_{33}$ 's for KDP are shown in Fig. 11. It is apparent that the $U_{33}$ of the hydrogen atom is larger than those of the other atoms because of the low mass of hydrogen. On the other hand, the potassium, phosphorus, and oxygen atoms show much smaller $U_{33}$ 's than the hydrogen atom. Although some scattering of data may be observed in the paraelectric phase, the temperature dependences are essentially in agreement with the previous report by Nelmes et $a{ }^{22)}$ It should be noted that the $U_{33}$ 's of heavy atoms show no noticeable changes through the paraelectric-ferroelectric phase transition. These values decrease monotonically with decreasing temperature.

Figure 12 shows the temperature dependences of the anisotropic atomic displacement parameters, $U_{33}$ 's, of DKDP. In the paraelectric phase, the temperature dependences are in good agreement with those shown by dotted lines. ${ }^{22)}$ Similarly to that observed in KDP, the potassium, phosphorus, and oxygen atoms show much smaller $U_{33}$ 's than the deuterium atom. It should be noted that the $U_{33}$ 's of all the atoms show no noticeable changes throughout the paraelectricferroelectric phase transition. Only the $U_{33}$ of the phosphorus atom may change rapidly at $T_{\mathrm{c}}$, although the data scattering prevents us from drawing a decisive conclusion.

Here, we mention the temperature dependences of $U_{11}$ and $U_{22}$. In the paraelectric phase, $U_{11}$ and $U_{22}$ decrease monotonically with decreasing temperature. As for the relative magni- 
tude, $U_{11}^{\mathrm{P}}<U_{11,22}^{\mathrm{O}}<U_{11}^{\mathrm{K}}<U_{11,22}^{\mathrm{H} / \mathrm{D}}$, where the superscripts indicate the atoms. Since $U_{33}^{\mathrm{K}}<U_{33}^{\mathrm{P}}<U_{33}^{\mathrm{O}}<U_{33}^{\mathrm{H} / \mathrm{D}}$, as shown in Figs. 11 and 12, the nuclear distribution of $\mathrm{K}$ is planular within the $x y$-plane, while that of $\mathrm{P}$ is elongated along the $z$ axis. The ratios of $\sqrt{U_{11} / U_{33}}$ at $T_{\mathrm{c}}$ are about $1.2,0.73$, and 0.78 for $\mathrm{K}, \mathrm{P}$, and $\mathrm{O}$, respectively. These values are almost in agreement with the previous reports. ${ }^{22,26)}$ Within the ferroelectric phase, the temperature variations of $U_{11}$ and $U_{22}$ are small and the values are almost continuous to the paraelectric ones.

\section{Discussion}

In the previous section, the interatomic distances and angles are explained in the figures. At low temperatures, the structures of KDP and DKDP are almost the same except for the cell parameter $a$. The parameter $a$ of DKDP is $0.5 \%$ $(0.05 \AA)$ larger than KDP, which reflects the difference in the hydrogen-bonded $\mathrm{O} 1-\mathrm{O} 2$ distance $R_{\mathrm{O}-\mathrm{O}}$; that of DKDP is about $0.03 \AA$ longer than of KDP. The magnitudes and temperature dependences of the atomic displacements along the polar axis perpendicular to hydrogen bonds are almost the same in both crystals. Another difference is the bond distance between $\mathrm{O}$ and $\mathrm{H} / \mathrm{D}$; in $\mathrm{KDP} \mathrm{O} 1-\mathrm{H}, r_{\mathrm{H}}=1.06 \AA$, while in DKDP O1-D, $r_{\mathrm{D}}=1.03 \AA$ in the ferroelectric phase. Even if a proton and a deuteron are within the same local potential, the zero-point energy depends on the particle mass. Therefore, the average position of a deuteron will be closer to $\mathrm{O} 1$ than that of a proton, which is in agreement with the abovementioned result.

Here, we comment on the 'precursor' temperature dependence of $\delta$ in KDP. As shown in Fig. 7, $\delta$ decreases with decreasing temperature in the paraelectric phase. If it was a precursor effect of the ferroelectric transition, $\delta$ would increase on approaching $T_{\mathrm{c}}$, since $r_{\mathrm{H}}$ decreases after the ferroelectric transition, as shown in Fig. 6. The parameter $\delta$ reflects the shape of the nuclear density, as determined by the split-atom method. The nuclear density will be given by the superposition of two wave functions of the ground and first-excited states. ${ }^{21)}$ Since the energy gap is large enough in KDP, the weight of the upper energy level decreases rapidly with decreasing temperature, so that $\delta$ decreases at lower temperatures. On the other hand, the energy gap is so small in DKDP that the $\delta$ of deuterons may not change with temperature.

Some authors claimed that the difference in $R_{\mathrm{O}-\mathrm{O}}$ causes an isotope effect on the transition temperature (geometrical isotope effect). However, they could not explain why $R_{\mathrm{O}-\mathrm{O}}$ differs. ${ }^{11-13)}$ We consider that the more rapid motion of protons than of deuterons bonds the two oxygen atoms more strongly, so that the geometrical isotope effect itself may be caused by the tunneling motion of protons. As a consequence of the short $R_{\mathrm{O}-\mathrm{O}}$, the $\delta$ of KDP is smaller than that of DKDP, and the localization of protons is prevented down to low temperatures. Under hydrostatic pressure, a small $R_{\mathrm{O}-\mathrm{O}}$ turns the double-minimum potential for protons/deuterons into a single-minimum type; finally, the transition temperatures disappear. $^{33)}$

If hydrogen localizes by $\mathrm{O} 1$ below $T_{\mathrm{c}}$, then the tetragonal $\mathrm{PO}_{4}{ }^{3-}$ ( site symmetry of $\mathrm{S}_{4}$ ) changes into $\mathrm{H}_{2} \mathrm{PO}_{4}{ }^{-}$( site symmetry of $C_{2}$ ), in which $\mathrm{P}$ shifts toward the $\mathrm{O} 2$ side and induces a dipole moment along the $c$-axis. Simultaneously, the $\mathrm{K}^{+}$ion
Table IV. Atomic displacement parameters $U_{33}$ 's [ $\left.\AA^{2}\right]$ of KDP and DKDP above and below $T_{\mathrm{c}}$. The split distances $d$ 's $[\AA]$ are assigned using the displacements in the ferroelectric phase. The calculated $U_{33}^{\text {Para }}$ is given by eq. (4) in the text.

\begin{tabular}{ccccc}
\hline $\begin{array}{c}\text { KDP } \\
\text { Atom }\end{array}$ & $d$ & $U_{33}(120 \mathrm{~K})$ & $U_{33}^{\text {Para }}$ & $U_{33}(150 \mathrm{~K})$ \\
\hline $\mathrm{K}$ & 0.0328 & 0.00853 & 0.00961 & 0.00647 \\
$\mathrm{P}$ & 0.0515 & 0.00941 & 0.0121 & 0.00912 \\
\hline \multicolumn{5}{c}{} \\
\hline DKDP & & $U_{33}(210 \mathrm{~K})$ & $U_{33}^{\text {Para }}$ & $U_{33}(220 \mathrm{~K})$ \\
\hline Atom & $d$ & 0.00648 & 0.00898 & 0.00730 \\
$\mathrm{~K}$ & 0.0500 & 0.00861 & 0.0136 & 0.0108 \\
\hline $\mathrm{P}$ & 0.0707 & & &
\end{tabular}

shifts to $\mathrm{P}$. The deformation of the $\mathrm{D}_{2} \mathrm{PO}_{4}{ }^{-}$molecule is similar to that of $\mathrm{H}_{2} \mathrm{PO}_{4}{ }^{-}$, except for the small difference in bond length between $\mathrm{O} 1-\mathrm{D}$ and $\mathrm{O} 1-\mathrm{H}$. The deformation of the molecule is considered to be induced by a change of chemical bonding. First-principles calculations have shown that some amount of electron charge is redistributed during the transition. $^{32)}$ Although neutron structural analysis can only determine the nuclear positions, the difference between $r_{\mathrm{H}}$ and $r_{\mathrm{D}}$ surely affects the amount of the spontaneous shift of $\mathrm{P}$ in the ferroelectric phase.

Some researchers claimed that the phosphate molecule was a disordered $\mathrm{H}_{2} \mathrm{PO}_{4}{ }^{-}$cluster even in the paraelectric phase. ${ }^{14-17)}$ If this is real, then the atomic displacement parameter $U_{33}^{\mathrm{Para}}$ of $\mathrm{P}$ or $\mathrm{K}$ in the paraelectric phase can be expressed using $U_{33}^{\text {Ferro }}$ in the ferroelectric phase as ${ }^{42)}$

$$
U_{33}^{\text {Para }}=d^{2}+U_{33}^{\text {Ferro }}
$$

where $2 d$ is the assigned split distance of $\mathrm{P}$ or K. Usually, the split distance is determined by structural analysis while assuming split atoms in the disordered phase. However, such analysis failed in both KDP and DKDP in our determination. The least-squares calculations converged in $d=0$; no splitting for K, P nor O. Here, we may estimate $d$ from the spontaneous displacements in the ferroelectric phase. As shown in Table $\mathrm{IV}$, the atomic displacement parameter $U_{33}$ of $\mathrm{K}$ is small in the paraelectric phase; therefore, eq. (4) does not hold at all. The $U_{33}$ 's of $\mathrm{O} 1$ and $\mathrm{O} 2$ decrease smoothly through $T_{\mathrm{c}}$, and do not obey eq. (4). Moreover, the oxygen atoms remain at almost the same $z$ 's from the center of gravity, throughout the phase transition.

It has been noted that the $U_{33}$ of $\mathrm{P}$ is larger than $U_{11}$ in the paraelectric phase, which is reconfirmed in our analyses. However, it changes smoothly at $T_{\mathrm{c}}$ in KDP, which does not support a finite $d$ in the paraelectric phase. On the other hand, the $U_{33}$ of $\mathrm{P}$ may decrease abruptly below $T_{\mathrm{c}}$ in the case of DKDP. From eq. (4), $d$ is estimated at about $0.014 \AA$, which is much smaller than the spontaneous displacement of $c a$. $0.07 \AA$. Therefore, we cannot conclude that the distorted $\mathrm{H}_{2} \mathrm{PO}_{4}{ }^{-}$cluster exists in the paraelectric phase from a structural point of view. It should be noted that eq. (4) holds if the transition mechanism is an order-disordered one, and the reverse is not true. Even if the transition mechanism is displacive, an atomic displacement parameter sometimes decreases steeply below $T_{\mathrm{c}}{ }^{42}$ )

From the figures in the last section, the structural change at 
$T_{\mathrm{c}}$ is discontinuous and is accompanied by a jump. Only in KDP, can a gradual change be recognized just below $T_{\mathrm{c}}$. This may be related to the residual double peaks of proton density in the ferroelectric phase, as reported by Nelmes et al. ${ }^{25)}$ With decreasing temperature below $T_{\mathrm{c}}$, the proton density changes gradually into the ground-state wave function form, so that the distortion of the $\mathrm{PO}_{4}$ tetrahedron and the shift of the $\mathrm{K}$ atom approach the values at $0 \mathrm{~K}$. In DKDP, the deuteron may behave as a more classical particle, and its nuclear density does not indicate a quasi-double-peak below $T_{\mathrm{c}}$.

\section{Conclusions}

In this report, we have refined the atomic parameters in both the paraelectric and ferroelectric phases, in order to elucidate the isotope effect of the structural difference between KDP and DKDP. The structures have been analyzed using neutron diffraction data from single crystals at ten temperatures down to $10 \mathrm{~K}$. The temperature dependences of the parameters have been discussed not from two or three temperature points but from ten data points in a wide temperature range. Here, we summarize the structural changes at the ferroelectric transition and the differences between KDP and DKDP.

(i) The refined structural parameters are in agreement with the previous results, in general. Protons/deuterons are in disorder in the paraelectric phase, while the potassium and phosphorus atoms are not in disorder in both KDP and DKDP, from the crystallographic viewpoints. In the ferroelectric phases, the displacements of the potassium and phosphorus atoms along $c$-axis arise, in connection with the ordering of protons/deuterons. These characteristics are essentially the same as the previous reports. $^{22-27)}$

(ii) In DKDP, the atomic coordinates show no remarkable temperature dependence except for the stepwise change during the ferroelectric transition. On the other hand, the 'precursor' behavior in the paraelectric phase and the continuous changes of the atomic coordinates just below $T_{\mathrm{c}}$ are confirmed in KDP.

(iii) The displacements of nuclei accompanied by the phase transition are considered in keeping a common center of gravity of the unit cell in both KDP and DKDP. In the ferroelectric phase, the potassium and phosphorus nuclei display remarkable displacements, and the oxygen and hydrogen nuclei tiny shifts along the polar axis, in comparison with those in the paraelectric phase. The antiparallel displacements of the potassium and phosphorus atoms keep the center of gravity of the unit cell throughout the phase transition.

(iv) The spontaneous polarizations along the $c$-axis are calculated from the structural parameters by the point-charge method $(\mathrm{K}:+e, \mathrm{P}:+5 e, \mathrm{O}:-2 e$, and $\mathrm{H} / \mathrm{D}:+e)$. The calculated magnitudes of the spontaneous polarization are in agreement with the previous values measured directly. The contribution of the atoms to the polarization mainly consists of one associated with the displacement of the phosphorus atom with a large valence charge. The experimental spontaneous polarization of DKDP is about 1.2 times larger than that of KDP, as reported previously, and this difference arises from the slightly larger displacement of the phosphorus atom in DKDP than in KDP. (v) In KDP, the atomic shifts along the $c$-axis indicate gradual increases with decreasing temperature just below $T_{\mathrm{c}}$. This character is consequently associated with the temperature dependence of the spontaneous polarization.

(vi) Except for the temperature dependence just below $T_{\mathrm{c}}$ in $\mathrm{KDP}$, no remarkable change is found in the crystal structure below the liquid-nitrogen temperature. At very low temperatures, the thermal contraction of the cell affects the magnitude of the displacements, i.e., the spontaneous polarizations, although the decreases are small.

(vii) All the atomic distances and angles are examined in both KDP and DKDP. From the result, remarkable differences are confirmed in the geometrical parameters relevant to the hydrogen bonds (O-H..O bond), while no significant difference is confirmed in the other parameters.

(viii) The separation distance between equilibrium sites of the hydrogen atom, $\delta$, is $c a$. $0.13 \AA$ longer in DKDP than in $\mathrm{KDP}$, and the distance between two oxygen atoms of the hydrogen bond, $R_{\mathrm{O}-\mathrm{O}}$, has a difference of about $0.04 \AA$ between KDP and DKDP. These two are reconfirmed in our study, and we additionally found that deuteron has a smaller distance from the acceptor oxygen atom; the distance $r_{\mathrm{D}}$ is about $0.04 \AA$ shorter than $r_{\mathrm{H}}$ in both the paraelectric and ferroelectric phases. $r_{\mathrm{D}}$ depends slightly on temperature, while $r_{\mathrm{H}}$ strongly.

Even if the proton and deuteron are located at the same potential, the remarkable difference should appear in their reciprocating motions between two equilibrium positions because of their mass. Consequently, it generates a difference in the strength of the hydrogen bond; if a proton vibrates more frequently, it makes the distance $R_{\mathrm{O}-\mathrm{O}}$ shorter. Therefore, the isotope effect for KDP and DKDP can be explained as a result of such a mass effect. Additionally, the difference between the behaviors of proton and deuteron is found to definitely increase the difference of the spontaneous polarizations; a heavy deuteron attaches closer to the acceptor O1, and the electron charge transfer from $\mathrm{O} 1$ to $\mathrm{D}$ takes place, so that the shift of the phosphorus atom can become larger.

Our structural analyses support a scenario in which the differences of the hydrogen mass and the potentials associated with different distances between the two oxygen atoms cause the differences in the transition temperature, the spontaneous polarization, and the vanishing of the ferroelectric phases at different critical pressures in KDP and DKDP. Namely, 'tunneling' hydrogen is the key to understanding the phase transition of KDP-type ferroelectrics, although 'tunneling' may be a dynamical disorder without quantum coherency. Finally, a disordered or distorted $\mathrm{H}_{2} \mathrm{PO}_{4}{ }^{-}$cluster in the paraelectric phase has not been established from the structural point of view.

\section{Acknowledgements}

We thank Dr. Kazuo Gesi for providing KDP single crystals of fine quality, and Dr. Hironobu Kasano for his advice on the growth of single crystal of DKDP and for his fruitful comments about chemical bonding. One of the authors (T. M.) received warm encouragements for writing this paper from Dr. Kiyoshi Shiraishi, Dr. Yukihiko Kawamura, and Mr. Naohiro Koshiji; he extends his grateful thanks to Dr. Hirotake Shigematsu for assistance in the experiment. 
The neutron diffraction experiments at JRR-3M were supported by the Institute for Solid State Physics (The University of Tokyo) and JAEA under experiment-project Nos. 6746, 7780, 8777, and 9736.

1) G. Busch and P. Scherrer: Naturwissenschaften 23 (1935) 737.

2) J. C. Slater: J. Chem. Phys. 9 (1941) 16.

3) W. Bantle and P. Scherrer: Nature 143 (1939) 980.

4) G. E. Bacon and R. S. Pease: Proc. R. Soc. London, Ser. A 220 (1953) 397.

5) G. E. Bacon and R. S. Pease: Proc. R. Soc. London, Ser. A 230 (1955) 359.

6) R. Blinc: J. Phys. Chem. Solids 13 (1960) 204.

7) P. G. de Gennes: Solid State Commun. 1 (1963) 132.

8) M. Tokunaga and T. Matsubara: Prog. Theor. Phys. 35 (1966) 581.

9) K. K. Kobayashi: J. Phys. Soc. Jpn. 24 (1968) 497.

10) E. Matsushita and T. Matsubara: Prog. Theor. Phys. 67 (1982) 1

11) M. Ichikawa: Acta Crystallogr., Sect. B 34 (1978) 2074.

12) M. Ichikawa: Chem. Phy. Lett. 79 (1981) 583

13) M. Ichikawa, K. Motida, and N. Yamada: Phys. Rev. B 36 (1987) 874

14) Y. Tominaga, M. Kasahara, H. Urabe, and I. Tatsuzaki: Solid State Commun. 47 (1983) 835

15) Y. Tominaga, H. Urabe, and M. Tokunaga: Solid State Commun. 48 (1983) 265.

16) Y. Tominaga, Y. Kawahata, and Y. Amo: Solid State Commun. 125 (2003) 419.

17) Y. Kawahata and Y. Tominaga: Solid State Commun. 145 (2008) 218

18) M. C. Lawrence and G. N. Robertson: J. Phys. C: Solid State Phys. 13 (1980) L1053.

19) H. Sugimoto and S. Ikeda: Phys. Rev. Lett. 67 (1991) 1306.

20) C. Totsuji and T. Matsubara: J. Phys. Soc. Jpn. 63 (1994) 2760.

21) H. Mashiyama: J. Korean Phys. Soc. 46 (2005) 63.

22) R. J. Nelmes, G. M. Meyer, and J. E. Tibballs: J. Phys. C: Solid State
Phys. 15 (1982) 59.

23) J. E. Tibballs, R. J. Nelmes, and G. J. McIntyre: J. Phys. C: Solid State Phys. 15 (1982) 37.

24) W. F. Kuhs, R. J. Nelmes, and J. E. Tibballs: J. Phys. C: Solid State Phys. 16 (1983) L1029.

25) R. J. Nelmes, W. F. Kuhs, C. J. Howard, J. E. Tibballs, and T. W. Ryan: J. Phys. C: Solid State Phys. 18 (1985) L711.

26) Z. Tun, R. J. Nelmes, W. F. Kuhs, and R. F. D. Stansfield: J. Phys. C: Solid State Phys. 21 (1988) 245.

27) R. J. Nelmes: J. Phys. C: Solid State Phys. 21 (1988) L881.

28) S. Ikeda, K. Shibata, Y. Nakai, and P. W. Stephens: J. Phys. Soc. Jpn. 61 (1992) 2619

29) G. A. Samara: Ferroelectrics 7 (1974) 221.

30) S. Endo, K. Deguchi, and M. Tokunaga: Phys. Rev. Lett. 88 (2002) 035503.

31) G. F. Reiter, J. Mayers, and P. Platzman: Phys. Rev. Lett. 89 (2002) 135505 .

32) S. Koval, J. Kohanoff, J. Lasave, G. Colizzi, and R. L. Migoni: Phys. Rev. B 71 (2005) 184102.

33) S. E. Mkam Tchouobiap and H. Mashiyama: Phys. Rev. B 76 (2007) 014101 .

34) Y. Noda, H. Kimura, R. Kiyanagi, A. Kojima, Y. Morii, N. Minakawa, and N. Takesue: Proc. 1st. Int. Symp. Advanced Science Research (ASR2000) “Advances in Neutron Scattering Research”, J. Phys. Soc. Jpn. Suppl. A 70 (2001) 456.

35) Profile Check, DABEXN, and RADIELN can be obtained via http: //www . tagen. tohoku. ac. jp/labo/noda/FONDER/FONDER. html.

36) P. Coppens, T. N. Guru Row, P. Leung, E. D. Stevens, P. J. Becker, and Y. W. Yang: Acta Crystallogr., Sect. A 35 (1979) 63.

37) G. M. Sheldrick: Acta Crystallogr., Sect. A 64 (2008) 112.

38) L. J. Farrugia: J. Appl. Crystallogr. 32 (1999) 837.

39) W. Cochran: Adv. Phys. 18 (1969) 157.

40) G. A. Samara: Ferroelectrics 5 (1973) 25.

41) Y. Uesu, T. Tanaka, and J. Kobayashi: Ferroelectrics 7 (1974) 247.

42) K. Itoh: J. Phys. Soc. Jpn. 53 (1984) 2049. 